\title{
Placeboeffekte in der Akutschmerztherapie
}

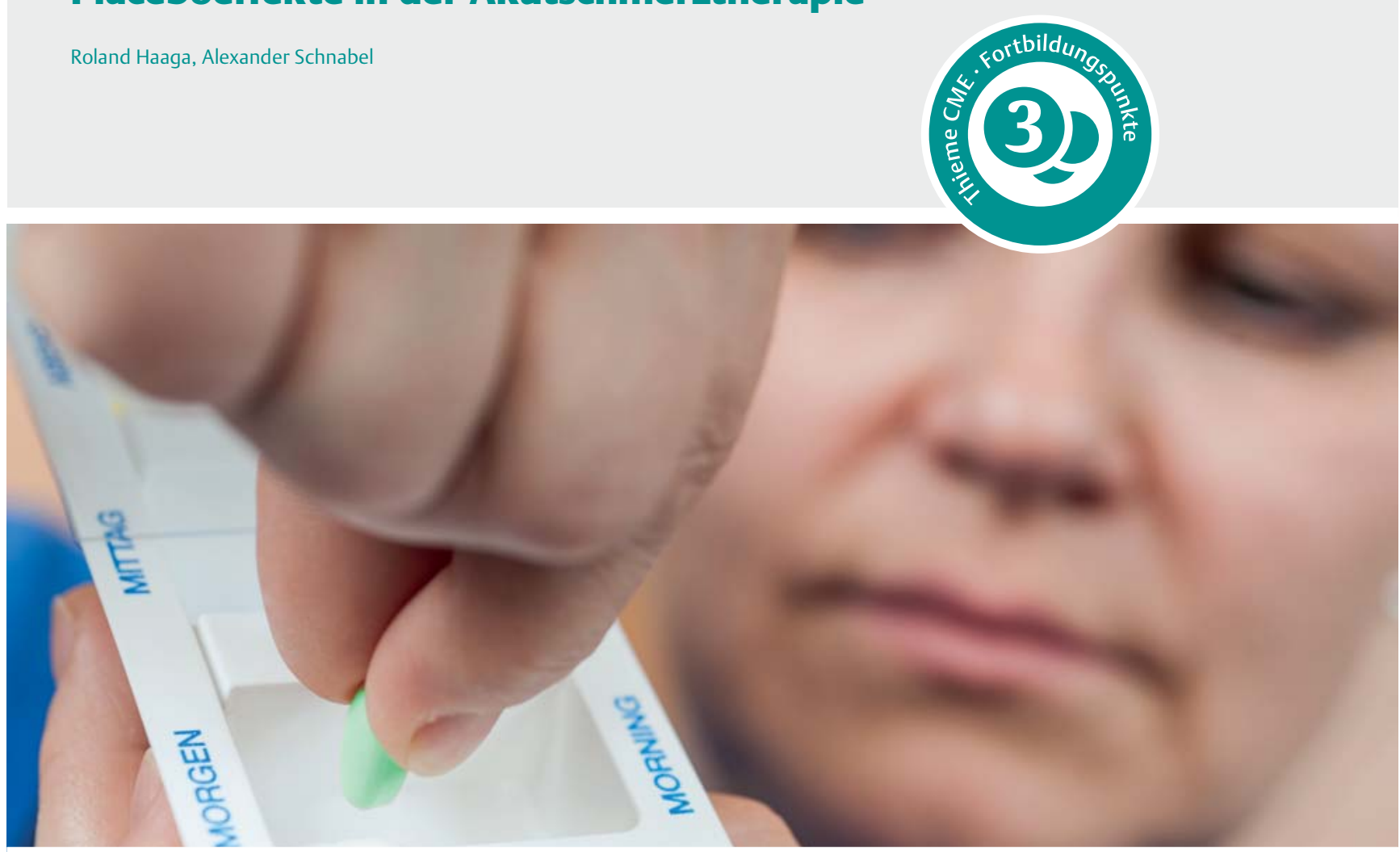

Quelle: KH Krauskopf.

\section{Placebo- und Noceboeffekte stehen schon lange im Fokus der Wissenschaft. In den letzten Jahren konnten wesentliche Erkenntnisse über die zugrunde liegenden Me- chanismen sowie potenzielle klinische Einsatzmöglichkeiten gewonnen werden. Dieser Beitrag gibt einen Überblick über den aktuellen Stand der Placeboanalgesie- Forschung, neuro- und psychophysiologische Zusammenhänge und die klinische Anwendung im Hinblick auf die Akutschmerztherapie.}

\section{Einleitung}

Ein wichtiges Ergebnis der Forschung der letzten Jahre ist: Es gibt nicht nur einen unspezifischen Placeboeffekt, sondern es wurden bis heute spezifische Mechanismen in 12 verschiedenen Körpersystemen nachgewiesen. Der analgetische Placeboeffekt spielt dabei eine entscheidende Rolle. Es wird diskutiert, wie diese Effekte gezielt klinisch anwendbar sind. Die bislang wenigen existierenden klinischen Studien mit Patienten zeigen signifikante schmerzlindernde Einflüsse analgetischer Placeboeffekte. Negative medikamentöse Effekte, die sog. algetischen Noceboeffekte, tragen ebenfalls einen entscheidenden Anteil zum Therapieerfolg bzw. -misserfolg bei.

\section{Relevanz von Placeboeffekten}

Placeboeffekte stehen in der Forschung und klinischen Praxis gegenwärtig sehr im Fokus der Diskussion. Beispielhaft sei hier die Untersuchung von Moseley und Kollegen zitiert. Sie zeigte, dass die vielfach durchgeführte Kniegelenksarthroskopie mit Débridement bei Gonarthrose einer Scheinoperation in den Endpunkten Schmerz und Funktion nicht überlegen war [1]. In einer aktuell veröffentlichten Studie von Beard und Kollegen wurden diese Ergebnisse für die arthroskopische subakromiale Dekompression bei schmerzhaftem Engpasssyndrom der Schulter bestätigt - auch wenn in dieser Studie keine Placebooperation (Simulation durch erfolgten Hautschnitt), sondern lediglich kein operativer Eingriff durchgeführt wurde [2]. 
Merke

Placeboeffekte sind demnach nicht nur im Rahmen medikamentöser Therapien, sondern auch bei interventionellen und operativen Therapien relevant.

In einem systematischen Review zeigten Wartolowska und Kollegen, dass $51 \%$ der untersuchten operativen bzw. endoskopischen Verfahren keinen Unterschied zwischen Placebo- und Interventionsgruppe aufwiesen. Zudem fiel der Unterschied bei den Verfahren, in denen die Intervention der Placebotherapie überlegen war, eher gering aus [3]. Anzumerken ist aber, dass im zitierten Review keine größeren chirurgischen Eingriffe, wie Laparotomien, Thorakotomien oder ausgedehnte Resektionen, berücksichtigt wurden. Auch wurde nicht bei allen dort eingeschlossenen Studien tatsächlich eine Placebooperation (z.B. Hautschnitt, aber keine aktive Behandlung) durchgeführt. Die Autoren schlussfolgerten, dass Operationen zwar nicht generell infrage gestellt werden dürfen. Sie empfahlen aber, auch bei operativen Therapien zukünftig placebokontrollierte Studien durchzuführen, um potenziell unnötige oder falsche Behandlungen zu vermeiden.

\section{Leitlinien}

In der Akutschmerztherapie wurde der analgetische Placeboeffekt erstmals in der 2007 veröffentlichten und 2009 aktualisierten S3-Leitlinie „Behandlung akuter perioperativer und posttraumatischer Schmerzen“ berücksichtigt $[4,5]$. Hier wurde empfohlen, dass der Placeboeffekt in der Schmerztherapie durch positive und realistische Informationen so weit wie möglich ausgeschöpft werden sollte. Ebenso soll der Noceboeffekt durch Vermeidung negativer oder angsterzeugender Informationen so weit wie möglich reduziert werden. Die Leitlinie wird derzeit überarbeitet und 2018 in aktualisierter Fassung erscheinen.

\section{Placebo- und Noceboeffekte}

Was versteht man unter analgetischen Placebo- und algetischen Noceboeffekten?

\section{DEFINITION}

Placebo

Als Placebo (lateinisch: „ich werde gefallen“) definiert der Duden ein Medikament, das einem echten Medikament in Aussehen und Geschmack gleicht, ohne dessen Wirkstoffe zu enthalten. Der Placeboeffekt bewirkt dann eine positive Veränderung im Körper aufgrund einer symbolischen Bedeutung, die einem Ereignis oder einem Objekt in einem heilenden Kontext zugeschrieben wird.
Die Placeboanalgesie tritt auf, wenn Veränderungen in der Schmerzwahrnehmung eintreten, die die pharmakologischen, psychologischen oder physiotherapeutischen Effekte einer Schmerzbehandlung übertreffen [6]. Sie ist damit also nicht auf den Einsatz von Scheinmedikamenten beschränkt, sondern umfasst tatsächlich alle Aspekte einer Schmerzbehandlung. So kann auch das reine ärztliche Gespräch mit Ausschöpfen der Regeln einer Kommunikation einen ausgeprägten Placeboeffekt generieren. Außerdem können auch pharmakologisch wirksame Medikamente einen sog. additiven, psychologischen Placeboeffekt erzeugen, der das rein pharmakologische Wirkspektrum erweitert [7]. Effekte der alternativen Medizin, wie z.B. Akupunktur, Homöopathie und Naturheilverfahren, beruhen zum Teil auch auf einer Placeboanalgesie [8]. Speziell für die Akupunktur liegen im Rahmen der GERAC-Trials (German Acupuncture Trials, 20022007, http://www.gerac.de) ausgezeichnete Daten vor (s. „Hintergrund - Akupunktur“).

\section{HINTERGRUND \\ Akupunktur \\ Die Daten der GERAC-Trials zeigen, dass Akupunktur bei chronischen Rücken- und Gonarthroseschmerzen der leitliniengerechten Therapie überlegen ist. Auf- grund dieser Daten wurde in Deutschland die Aku- punktur durch den Gemeinsamen Bundesausschuss zur Kassenleistung ernannt. Im Rahmen der GERAC- Trials machte es jedoch keinen Unterschied hinsicht- lich des Behandlungserfolgs, ob eine Verumaku- punktur nach den Empfehlungen der traditionellen chinesischen Medizin oder eine sog. Sham-Akupunk- tur (zufällige Verteilung der Nadelpositionen) durch- geführt wurde. Somit könnte ein Placeboeffekt eine Rolle spielen. Die Studienlage hierzu ist insgesamt jedoch nicht eindeutig, wie kürzlich in einer Meta- analyse von Vickers und Kollegen gezeigt werden konnte [9].}

Eine Schmerzlinderung nach Einnahme eines Placebos sagt jedoch noch nichts über die wahre Wirksamkeit des Placebos aus. Die Schmerzlinderung könnte Produkt des natürlichen Krankheitsverlaufs oder einer Spontanheilung sein. Will man einen wahren Effektivitätsnachweis einer Placebobehandlung - also einen Placeboeffekt aufzeigen, ist der Vergleich mit einer dritten Gruppe (einer sog. Natural History Gruppe [NH]) erforderlich, die nicht behandelt wurde. Die signifikante Schmerzreduktion durch die Placebobehandlung im Vergleich zu dieser $\mathrm{NH}$-Gruppe wird als wahrer Placeboeffekt interpretiert.

Während der Placeboeffekt prinzipiell wünschenswerte Effekte bewirkt, wie z. B. Schmerzreduktion oder Verbesserung der Beweglichkeit, kommt es im Rahmen eines Noceboeffektes umgekehrt zu unerwünschten Wirkun- 
gen. Dies kann sich sowohl als Symptomverschlechterung (Schmerzzunahme, zunehmende Immobilität) wie auch als Arzneimittelnebenwirkung, wie z.B. Übelkeit oder Hitzegefühl, äußern.

\section{Merke}

Placebo- und Noceboeffekte sind prinzipiell ubiquitär vorhanden und nicht auf einzelne Symptomentitäten beschränkt.

So sind relevante Placeboeffekte auch bei Morbus Parkinson, Übelkeit, Depression, kognitiver Leistung, vegetativer Aktivität und selbst bei der Immunregulation beschrieben.

\section{Wo entsteht der Placeboeffekt?}

Analgetische Placeboeffekte sind nicht nur subjektives Erleben. In zahlreichen Studien konnte gezeigt werden, dass ein Zusammenhang mit dem Endorphinsystem besteht [10-14]. Bereits 1978 konnte durch Levine und Kollegen gezeigt werden, dass sich placeboanalgetische Effekte durch die Gabe des Opiatrezeptorantagonisten Naloxon größtenteils aufheben lassen. Die Autoren folgerten, dass Placeboanalgesie über die Ausschüttung endogener Opioide vermittelt wird [14].

Bei vielen Sinnesleistungen, wie z. B. dem Hören oder Sehen, existiert ein klar hierarchisch gegliedertes neuronales Netzwerk mit einem primären sensorischen Kortex. Dahingegen gibt es in der Schmerzwahrnehmung nicht den einen Schmerzkortex. Große Fortschritte wurden in den letzten Jahren mithilfe der funktionellen Magnetresonanztomografie (fMRT) gemacht. Die fMRT ist ein bildgebendes Verfahren, das es ermöglicht, physiologische Funktionen u.a. im Bereich des zentralen Nervensystems darzustellen. Im Rahmen der Schmerzverarbeitung scheint ein äußerst komplexes Netzwerk aus interagierenden Arealen zu existieren, welche zum Teil auch multifunktional arbeiten. Die koordinierte Aktivierung dieser Areale ist relativ spezifisch für einen somatischen Schmerz, bildet aber wiederum keine seelischen Schmerzen ab [15].

Analog zur Erforschung der schmerzverarbeitenden Systeme wurde auch die Placeboanalgesie mittels fMRT und Positronenemissionstomografie (PET) untersucht. Zugrunde liegende Mechanismen spielen sich dabei in ähnlichen Hirnregionen ab wie bei echten Analgetika. Eippert und Kollegen zeigten, dass eine Placeboanalgesie durch Erwartung und Lernprozesse aufbaubar ist und durch Naloxon fast vollständig aufgehoben werden kann [13]. Placebos und Opioide wirken über dasselbe Netzwerk des opioidergen, absteigenden schmerzmodulierenden Systems. Placeboanalgesie führt zu reduzierter Hirnaktivität in schmerzsensitiven Hirnregionen (Thalamus, Insula, anteriorer zingulärer Kortex [ACC]) und es kommt zur Aus- schüttung endogener Opioide. Die Placeboanalgesie aktiviert das deszendierende, schmerzhemmende System und während der Erwartung des Schmerzes kommt es zur erhöhten Aktivität im präfrontalen Kortex - dies führt zu einer wahren Veränderung der Schmerzerfahrung [10, 16]. Insbesondere der ventromediale präfrontale Kortex scheint die zentrale Kernstruktur und Mediator im neuronalen Placebonetzwerk zu sein. Effekte des absteigenden schmerzmodulierenden Systems lassen sich unter Placeboanalgesie bis zur spinalen Ebene darstellen [13].

Merke

Placeboeffekte lassen sich im gesamten zentralen Nervensystem von der kortikalen bis hinunter zur spinalen Ebene nachweisen und werden über endogene Opioide und entsprechende Rezeptoren vermittelt.

Dieselben Strukturen sind auch bei Noceboeffekten, wie noceboinduzierter Hyperalgesie, involviert [17]. Letztlich kann man also davon ausgehen, dass ein Placebo das Verum tatsächlich auf neuronaler Ebene teilweise ersetzen oder dessen Effekte verstärken kann [18]. Andererseits kann ein Nocebo entsprechend die Verumwirkung abschwächen, aufheben oder die Wirkung ins Gegenteil umschlagen lassen [19].

\section{Wie entsteht der Placeboeffekt?}

Die dem Placeboeffekt zugrunde liegenden Mechanismen wurden in den letzten Jahrzehnten sehr intensiv erforscht. Im Zentrum der Betrachtung steht die Erwartungshaltung eines Patienten, der bei der Entstehung des Placeboeffektes eine bedeutende Rolle zugeschrieben wird. Sie wird über 3 psychologische Mechanismen vermittelt bzw. moduliert:

- Lernen inklusive der klassischen Konditionierung

- soziales Lernen inklusive des beobachtenden Lernens

- Kognition inklusive der Erwartungstheorie

\section{Klassische Konditionierung}

Bei der klassischen Konditionierung wird vorausgesetzt: Eine natürliche, unbedingte Reaktion kann durch eine erlernte, bedingte Reaktion ausgelöst werden, wenn diese wiederholt wird und in Assoziation aufgetreten ist. Der Mensch lernt, dass 2 eigentlich unabhängige Ereignisse regelmäßig miteinander verknüpft sind. Nach Einnahme eines Analgetikums kommt es zur Schmerzreduktion. Wird das Analgetikum durch ein wirkstofffreies, aber sonst identisches Placebo ersetzt, kann es zu einer ähnlichen Schmerzreduktion kommen. Das Placebopräparat ist zum konditionierten Stimulus geworden.

Analog gilt dies nicht nur für Placebopräparate, sondern wie oben bereits erwähnt - auch für andere, zuvor erfahrene und als wirksam angesehene Therapien. Die klassische Konditionierung spielt auch beim Noceboeffekt eine 
Rolle. Hier scheint es sogar so, dass sich eine Nocebohyperalgesie schneller als eine Placeboanalgesie konsolidieren lässt [20].

\section{Soziales Lernen und Beobachtungslernen}

Auch Prozesse des sozialen Lernens spielen bei der Ausbildung von Placeboeffekten eine bedeutende Rolle [21]. Die Beobachtung eines positiven Behandlungseffektes an einer anderen Person erzeugt bei dem Beobachter eine substanzielle Placeboanalgesie. Diese ist wiederum mit der Ausprägung von Empathie korreliert. Beobachtungslernen produziert Placeboeffekte, die genauso hoch sind wie die Effekte, die durch Konditionierung erzeugt werden. Placeboeffekte durch Erwartung sind signifikant geringer.

\section{Kognitives Modell inklusive Erwartungstheorie}

Die Erwartungstheorie geht davon aus, dass das, was ein Patient erwartet, auch eintritt. Im Rahmen klinischer Studien wird durch gezielte verbale Instruktion (z. B. „sehr starkes, neuartiges Schmerzmittel“) die Erwartung des Probanden beeinflusst. Analog lässt sich dies somit auch auf das Arzt-Patienten-Gespräch übertragen. Hier kann der Arzt im Gespräch die Erwartung des Patienten steuern, um dadurch den Placeboeffekt auch bei einem pharmakologischen Verum voll auszunutzen (s. „Hintergrund - Information und Vorerfahrung“).

\section{HINTERGRUND \\ Information und Vorerfahrung}

Müller und Kollegen zeigten, dass verbale Information alleine einen ausgeprägten Placeboanalgesieeffekt induzieren kann [22]. Eine bei der Hälfte der Probanden durchgeführte Konditionierungsprozedur hatte jedoch keinen signifikanten zusätzlichen Effekt. Erstaunlicherweise zeigte sich bei Placeborespondern mit anamnestisch schlechten Erfahrungen hinsichtlich schmerztherapeutischer Maßnahmen eine stärker ausgeprägte Placeboanalgesie als bei Patienten mit guten Erfahrungen. Die Autoren vermuten, dass gerade Patienten mit mehrfachem Therapieversagen in der Anamnese am meisten von einem neuen, potenziell besseren Therapieansatz profitieren. Im Gegensatz hierzu waren jedoch experimentell induzierte gute Erfahrungen auch im Verlauf mit besserer Placeboanalgesie verknüpft als bei schlechten Erfahrungen [23].

Dass Erwartung auch bei anderen Krankheiten einen signifikanten Unterschied im Therapieerfolg nach sich ziehen kann, zeigt sich exemplarisch beim Morbus Parkinson. Hier konnten Placebos zu einer vermehrten Ausschüttung von Dopamin im Striatum führen. Die Effektstärke zeigte sich wiederum abhängig von der Erwartung des Patienten an eine klinische Besserung [24].
Übereinstimmend kann man aus allen Untersuchungen folgern, dass Vorerfahrungen und Lernen einen relevanten Einfluss auf die Ausprägung der Placeboantwort haben.

\section{Merke \\ Bei der Generierung von Placeboeffekten spielen all- gemeine Lernprozesse, klassische Konditionierung und Erwartungsprozesse durch Instruktion eine Rol- le. Vor allem die kognitiven Neurowissenschaften be- trachten die Patientenerwartung als Hauptfaktor.}

\section{Weitere Einflussfaktoren}

\section{Wertbeimessung}

Sehr interessant ist, dass allein der Unterschied, ob ein Verum oder Placebo als teuer oder günstig deklariert wird, deutlichen Einfluss auf die Wirksamkeit hat. Das teure Placebo erzielt einen besseren analgetischen Effekt als die günstige Variante [25]. Analog gilt dies auch für Noceboeffekte [26]. Die Wertbeimessung entscheidet also u. a. über die Effektivität einer Maßnahme. Der Wert an sich ist dabei nicht nur über den finanziellen Wert zu bestimmen und auch nicht als absoluter Faktor zu erachten. So kann die Wertbeimessung situationsabhängig stark variieren.

Im Rahmen der postoperativen Akutschmerztherapie legen Patienten allgemein einen hohen Wert darauf, wenig Schmerzen zu haben. Man kann jedoch davon ausgehen, dass unterschiedliche Einstellungen bei den Patienten bestehen. Haben Patienten bereits schlechte Erfahrungen mit hochpotenten Opioiden gemacht (z. B. Nebenwirkungen, nicht opioidsensibler Schmerz oder schlicht zu niedrig dosiert), werden sie einem Opioid einen geringeren Wert beimessen als Patienten mit guten Erfahrungen. Außerdem kann man davon ausgehen: Viele Patienten (und natürlich auch Therapeuten) werden einem moderneren, synthetischen Opioid (z.B. Hydromorphon oder Tapentadol) einen höheren Wert beimessen als einem älteren Wirkstoff (z. B. Morphin) - sie versprechen sich vom neuen Präparat eine bessere Effektivität oder reduzierte Nebenwirkungen. Ähnliches gilt auch für die Anwendung technischer Geräte (z.B. Tropfenzähler vs. Infusomat) (s. „Fallbeispiel - TENS-Gerät“).

Inwiefern die Schmerzreduktion hier wirklich auf der postulierten Wirkungsweise des TENS-Gerätes oder doch eher auf einem Placeboeffekt beruht, lässt sich in diesem Einzelfall sicherlich nicht differenzieren. Dies ist das Ziel der Studie, in der die Schmerzreduktion mittels TENS-Gerät und Placebo-TENS (optisch nicht zu unterscheidendes, aber nicht funktionierendes Gerät) verglichen wird. Placeboeffekte könnten z.B. auf höheren Erwartungen an das TENS-Gerät als an Medikamente bzw. höherer Wertbeimessung an ein technisches Gerät mit direktem 


\section{FALLBEISPIEL}

\section{TENS-Gerät}

Eine 33-jährige Patientin wird im Kreißsaal nach erfolgter Kaiserschnittentbindung in Spinalanästhesie durch das Team der Geburtshilfe versorgt. Im postoperativen Verlauf beklagt die Patientin im Bereich des Unterbauchs ziehende Schmerzen mit einer Schmerzstärke von 10 Punkten auf einer numerischen Rangskala (NRS; 0 = kein Schmerz; 10 = stärkster vorstellbarer Schmerz). Die Patientin wirkt äußerst unruhig und ängstlich. Trotz der üblichen konservativen Therapie (Gabe von i. v. Piritramid sowie Paracetamol) lässt sich keine suffiziente Schmerzlinderung erzielen. Im Rahmen einer aktuell in Planung befindlichen Studie erhält die Patientin dann ein TENS-Gerät. Im Verlauf weniger Minuten nach Aufkleben der Elektroden und Beginn der Stimulation gibt die Patientin eine deutliche Schmerzlinderung auf NRS 5 an. Zusätzlich zeigt sich die Patientin jetzt deutlich entspannter und ruhiger.

Therapieansatz am schmerzhaften Bereich beruhen. Weiterhin können Sicherheit generiert und Ängste reduziert werden, indem die Patientin die Therapie mittels TENS selbst steuern kann.

Für den Schmerztherapeuten kann es somit insgesamt sehr sinnvoll sein, genau zu eruieren, was der Patient von den durchgeführten Maßnahmen bzw. der angeordneten Medikation erwartet. So lassen sich ggf. falsche Einschätzungen seitens des Patienten möglichst korrigieren.

\section{Merke}

Die Effektstärke hängt auch vom beigemessenen Wert ab. Dieser kann situationsabhängig stark variieren und damit auch schwankende Placeboeffektstärken begründen.

\section{Placebomerkmale und Persönlichkeitsstruktur}

In den meisten Studien zur Placeboanalgesie lässt sich mittels Placebo eine Schmerzreduktion um mindestens 20-30\% erreichen [27]. Hier stellt sich die Frage, wie man auch im klinischen Alltag einen maximalen Effekt erreichen kann. Allgemein bekannt ist, dass Injektionen besser wirken als dasselbe Präparat als Tablette. Auch andere Präparateeigenschaften (Farbe, Geruch, Größe, Form etc.) haben Einfluss. Aber nicht nur das Präparat selbst, sondern auch das Behandlungsumfeld, Patientenund Behandlereigenschaften können hochrelevant sein $(\vee$ Tab. 1).

Generell gibt es große interindividuelle Unterschiede in Bezug auf die jeweilige Empfänglichkeit gegenüber Placeboeffekten. Während manche Patienten sehr gut auf Placebo reagieren (sog. Placeboresponder), gibt es auch Patienten (z. B. mit narzisstischer oder histrionischer Persönlichkeit), die hierfür kaum bis gar nicht empfindlich sind (Nonresponder). Hierbei sind die zugrunde liegenden Charaktereigenschaften des Patienten relevant. Bei Placeborespondern sind in der Regel Eigenschaften wie Neurotizismus, primäre Suggestibilität, Submission und Optimismus stärker ausgeprägt als bei Nonrespondern. Außerdem sind weniger ängstliche Patienten meist empfänglicher für Placeboeffekte. Auch die Persönlichkeit des Therapeuten, insbesondere dessen Suggestivität, kann Auswirkungen auf die Stärke des Placeboeffektes haben.

Allerdings zeigen diverse Untersuchungen, dass die Placeboreagibilität zusätzlich auch situationsabhängig ist und dementsprechend auch intraindividuell stark variieren kann. So ist z. B. davon auszugehen: Ein sicher und seriös auftretender, erfahrener Ober- oder Chefarzt kann in einem ruhigen Einzelgespräch einen stärkeren Placeboeffekt generieren als ein sehr junger und womöglich unsicher wirkender Assistenzarzt am Patientenbett im Mehrbettzimmer.

\section{Offene vs. verdeckte Medikamentengabe}

Medikamente können so verabreicht werden, dass Patienten dies nicht bemerken. So kann das Zuspritzen eines Medikaments in eine liegende Infusionsleitung vom Patienten unbemerkt durchgeführt werden. Diese Medikamentenapplikation wird als verdeckte Gabe (engl.: hidden medication) bezeichnet. Die offene Gabe (engl.: open medication) erfolgt hingegen so, dass der Patient die Applikation bewusst wahrnimmt und darüber aufgeklärt wird, welches Medikament er gerade ver-

- Tab. 1 Wirkfaktoren und Faktoren für eine bessere Wirkung eines Placebos [5].

besondere Wirkfaktoren für die Effektivität der Placeboanalgesie

- Merkmale des Präparates bzw. der Behandlung (z. B. Farbe, Applikationsform)

- Merkmale des Patienten (z. B. Persönlichkeit, Vorerfahrungen)

- Merkmale des Behandlers und der therapeutischen Beziehung

- Merkmale des Behandlungskontexts (z. B. ambulant)

\section{Faktoren für eine bessere Placebowirkung}

- gleiche Verabreichungsform bzw. Aussehen wie das Verum

- sehr große bzw. kleine Größe der Tablette

- invasive Verabreichung (z. B. Injektion)

- bitterer, unangenehmer Geschmack

- farbiges Aussehen (am besten rot für Analgesie)

- Ausgabe in größerer Anzahl 
abreicht bekommt. Amanzio und Kollegen zeigten u.a., dass die Effektstärke eines pharmakologisch wirksamen Medikaments bei verdeckter Gabe signifikant geringer war als bei offener Gabe. Unter anderem wurden Metamizol, Buprenorphin und Tramadol untersucht. Die verdeckte Gabe ohne Wissen des Patienten schaltet somit den additiven Placeboeffekt des Medikaments aus. Bei gleicher Medikamentendosis wird daher ein deutlich geringerer Effekt erzielt [28].

Die Nutzung der sog. „open medication“ ist also Voraussetzung für die Generierung eines additiven Placeboeffektes. Dieses Prinzip basiert auf dem Grundgedanken, dass sich jedes Analgetikum einerseits aus einer pharmakologischen, andererseits aus einer psychologischen (kognitiv getriggerten) Komponente, dem Placeboeffekt, zusammensetzt. Offen verabreichte Analgetika, die der Patient bewusst wahrnimmt (z. B. eine intravenöse Injektion von Piritramid), haben einen besseren Effekt als Analgetika, die verdeckt verabreicht werden. Dies sind z. B. Opioide in einem Perfusor oder orale Schmerzmedikamente, die zusammen mit anderen Tabletten (z.B. Blutdruck) in der gleichen Tablettenschachtel liegen [7, 29].

Merke

Je besser ein Medikament wahrgenommen wird (Geschmack, Geruch, Aussehen, Berührung, Information), desto besser kann der Placeboeffekt ausgenutzt werden.

\section{Wie kann man im klinischen Alltag den Placeboeffekt nutzen?}

Im Rahmen wissenschaftlicher Studien lässt sich bei entsprechendem Studiendesign (NH-Gruppe, s. Abschnitt „Placebo- und Noceboeffekte“) ein ausgesprochen hoher Placeboeffekt generieren. Zugrunde liegen dabei jedoch zum Teil aufwendige Versuchsanordnungen und eine relativ intensive Arzt-Patienten-Beziehung mit Einzelbetreuung. Im klinischen Alltag fehlt aber regelmäßig die Zeit, sich ausführlich mit einem einzelnen Patienten zu befassen. Außerdem werden Therapien oft im Rahmen von Behandlungsstandards - sprich Standard Operating Procedures (SOP) - durchgeführt, sodass individuelle Konzepte im klinischen Alltag schwieriger umsetzbar sind.

Nichtsdestotrotz liegt hier natürlich das Potenzial für einen gut organisierten und fest im Klinikalltag verankerten Akutschmerzdienst. Hier kann im Rahmen von idealerweise täglichen Visiten durch spezialisiertes ärztliches und pflegerisches Personal (insbesondere Pain Nurses) ein individuelles und optimiertes Therapieregime erarbeitet werden. Die Akutschmerzdienste bedienen sich neben einer optimalen Verumtherapie auch des Placeboeffektes und können diesen bewusst und elegant im All- tag einsetzen, um seine additiven Effekte auszuschöpfen. Dies soll anhand des Fallbeispiels „Postoperative Schmerztherapie“ demonstriert werden.

\section{FALLBEISPIEL}

\section{Postoperative Schmerztherapie}

Eine 62-jährige Frau befindet sich nach Hysteroskopie im Aufwachraum. Sie klagt trotz erfolgter kumulativer i. v. Applikation von $15 \mathrm{mg}$ Piritramid, $1 \mathrm{~g}$ Metamizol und $1 \mathrm{~g}$ Paracetamol weiterhin über starke Schmerzen im Bauchraum, weniger im Operationsbereich. Die Patientin leidet unter chronischen Rückenschmerzen und erhält Tilidin/Naloxon $200 \mathrm{mg}$ retard pro Tag. Im Rahmen eines Gespräches, in dem der Patientin vermittelt wird, dass man ihre Schmerzen ernst nimmt, werden nach Ankündigung nochmals $7 \mathrm{mg}$ Piritramid appliziert. Im weiteren einfühlsamen und wertschätzenden Gespräch wird deutlich: Die Patientin ist aktuell aufgrund einer früheren Gewalterfahrung depressiv verstimmt und hat zurzeit keinen Schmerztherapeuten, der sie betreut. Es wird ihr angeboten, sich zeitnah in der Schmerzambulanz des Interdisziplinären Schmerzzentrums vorzustellen. Am Ende des Gespräches gibt die Patientin eine Schmerzreduktion von $70 \%$ an.

\section{Offene Gabe der Medikation}

Besonders im perioperativen Bereich bekommen Patienten regelmäßig Schmerzmittel verordnet. Bei zumeist oraler Applikation liegen diese allerdings oft mit mehreren anderen Tabletten zusammen in der Tablettenschachtel. Dem Patienten ist dann nicht unbedingt bewusst, dass bzw. wie oft und wann er ein Analgetikum bekommt. Diese Art der Verabreichung kann man also als verdeckte Gabe verstehen, die - wie oben beschrieben mit einer geringeren Wirkung verbunden ist. Analog gilt das natürlich auch für das unkommentierte Anhängen von Infusionen, i. v. Bolusgaben oder Dauerinfusion über Spritzenpumpen. Anzunehmen ist, dass Patienten diesen Maßnahmen bezüglich der Schmerztherapie weniger Wert beimessen, da diese nicht als eigentlich analgetisch wirksam erkannt werden.

Ziel sollte daher die offene Medikation sowie die Einbindung (engl.: „shared decision making“) des Patienten in die Auswahl der Medikamente (z.B. Opioide vs. Nichtopioide vs. Kombination) sein. Dem Patienten muss bewusst sein bzw. bewusst gemacht werden, dass und wann er ein Analgetikum erhält und welche Nebenwirkungen ggf. zu erwarten sind. Um dies im Stationsalltag und bei regelmäßiger Verordnung zu erreichen, gibt es mehrere Möglichkeiten, die unter „Praxis - Offene Medikation im Alltag“ dargestellt sind. 


\section{PRAXIS}

Offene Medikation im Alltag

- Dem Patienten werden initial die einzelnen Medikamente und speziell das Analgetikum gezeigt, sodass er die entsprechenden Tabletten dann im Verlauf auch selbst erkennt.

- Optimalerweise erfolgt die Erläuterung der analgetischen Medikation im Rahmen eines Schmerzkonsils durch einen Schmerztherapeuten oder eine versierte Pain Nurse.

- Der Patient erhält einen tagesaktuellen Medikationsplan mit entsprechenden Einnahmezeitpunkten.

- Das Analgetikum wird in einer gesonderten Verpackung angereicht.

- Das Analgetikum wird jeweils persönlich durch Arzt oder Pflegekraft angereicht.

- Neben der initiierten analgetischen Dauermedikation wird dem Patienten die Möglichkeit einer Bedarfsmedikation eingeräumt, die er sich selbstständig und damit sehr bewusst abfordern kann. Hier mag auch ein Vorteil der patientenkontrollierten Analgesie (PCA) liegen: Die Medikation wird durch den Patienten selbst gesteuert und erfolgt damit sehr bewusst. Eine PCA-Pumpe vermittelt ein Gefühl der Selbstkontrolle und der Sicherheit. Das Wissen, dass ein Analgetikum jederzeit verfügbar ist, kann Ängste reduzieren und damit bereits zu einem reduzierten Analgetikabedarf führen.

\section{Merke}

Ohne bewusste Medikamenteneinnahme fehlt der additive Placeboeffekt, was den Therapieerfolg abschwächen kann. Im schlechtesten Fall kann sogar ein negativer Effekt auftreten: Nebenwirkungen werden stärker wahrgenommen als die erwünschte Wirkung.

\section{Wahrnehmung schärfen}

Im Sinne der klassischen Konditionierung kann es sinnvoll sein, dem zunächst neutralen Stimulus mehr Aufmerksamkeit zu verschaffen. Der Patient soll sich also bei der Einnahme auf Farbe, Aussehen, Textur, Geschmack oder andere Präparateeigenschaften konzentrieren. Dies führt zur verstärkten und schnelleren Ausbildung eines bedingten Stimulus. Außerdem muss sich der Patient in der Folge dann auch den erzielten analgetischen Effekt, also den primär unbedingten Effekt, bewusst machen.

Das Problem dabei ist: Sind Schmerzen vorhanden, sind diese sehr präsent. Verschwinden die Schmerzen jedoch, fehlt der sensorische Input. Die Schmerzfreiheit oder Schmerzreduktion wird oft nicht bewusst wahrgenommen und der eigentliche Behandlungserfolg damit nicht „erlebt“.
Nimmt der Patient das Analgetikum nicht selbst ein, sondern bekommt es verabreicht, muss dies dann klar kommuniziert und idealerweise auch vor den Augen des Patienten „zelebriert“ werden. Dies gilt z. B. für die Situation unmittelbar postoperativ im Aufwachraum. Auch hier lohnt sich die Nachfrage nach der erfahrenen Schmerzreduktion, um damit die Effektivität der Therapie dem Patienten zu verdeutlichen. Relevant ist dabei auch, den Patienten zur Benutzung einfacher Intensitätsskalen, wie der bekannten NRS anzuleiten, damit er seine Schmerzintensität und entsprechende -reduktion besser einzuschätzen weiß.

\section{Aufklärung, Erwartung und Kommunikation}

Im Rahmen der Aufklärung lässt sich die Erwartung eines Patienten gezielt beeinflussen. Patienten, die bereits präoperativ eine hohe Schmerzerwartung und ein hohes Disstress-Niveau haben, geben postoperativ tatsächlich deutlich höhere Schmerzintensitäten an und haben einen entsprechend größeren Analgetikabedarf [30]. Entsprechend sollte bei sorgfältiger Aufklärung über ein zuverlässig wirksames postoperatives Schmerzmanagement die Erwartung des Patienten zum Positiven verändert werden. Je höher die Erwartung an eine suffiziente Analgesie, desto stärker ausgeprägt sollte wiederum auch der Placeboeffekt sein. Im perioperativen Bereich können Patienten dementsprechend bereits präoperativ, z. B. im Rahmen der anästhesiologischen Aufklärung, über das postoperative analgetische Therapieregime aufgeklärt werden. Hier kann auch darüber informiert werden, dass z. B. Analgetika anhand etablierter SOP auch ohne spezielle ärztliche Anordnung durch das Pflegepersonal verabreicht werden können. Somit lässt sich Sicherheit vermitteln und die Erwartung der Patienten verbessern.

\section{PRAXIS}

Chronische Schmerzen

Insbesondere bei Patienten mit chronischen Schmerzen kann es hilfreich sein, bereits präoperativ ein Schmerzkonsil durchzuführen. Hierbei kann man ein entsprechendes Analgesiekonzept vereinbaren und die Kollegen der behandelnden Fachdisziplin instruieren. Außerdem sollten diese Patienten nach der Operation durch den Akutschmerzdienst engmaschig visitiert und bei Bedarf und Patientenwunsch frühzeitig nach Entlassung an einen chronischen Schmerzdienst angebunden werden.

Auch wenn die Aufklärung der Patienten bezüglich des zu erwartenden Schmerzniveaus möglichst positiv sein sollte, dürfen andererseits keine unrealistischen Ziele versprochen werden. Die Glaubwürdigkeit der Präparate, der schmerztherapeutischen Interventionen (z. B. Regionalanästhesieverfahren) sowie des Therapeuten gilt es ebenso zu erhalten. Falsche Versprechungen werden in 
der Regel schnell entlarvt und machen den Behandelnden und seine Therapie unglaubwürdig. Unerwünschte Nebenwirkungen sollten dabei ebenfalls explizit erwähnt werden, ohne diese jedoch zu sehr zu betonen. Einerseits bekräftigen tatsächlich auftretende Nebenwirkungen die Wirksamkeit und damit auch den gewünschten Effekt einer Maßnahme, andererseits steigert dies noch einmal zusätzlich die Glaubwürdigkeit des Behandlers.

\section{Merke}

Im Rahmen der Aufklärung sind Glaubwürdigkeit, ein patientenzentriertes Vorgehen, kommunikative

Fähigkeiten und Fingerspitzengefühl gefragt.

Die Schwierigkeit besteht natürlich darin, unerwünschte Noceboeffekte zu vermeiden. Die zunehmende - größtenteils juristisch bedingte - Ausweitung der Aufklärung hin zu potenziellen, schweren unerwünschten Nebenwirkungen birgt die Gefahr, die Aufklärung als Aufzählung negativer Effekte zu gestalten - und damit Noceboeffekte zu generieren. Die eigentliche Indikation und die erwünschte Wirkung einer Maßnahme kommen dann leider oft zu kurz. So konnten Daniels und Kollegen zeigen: Nach intensiver Aufklärung über potenzielle postpunktionelle Kopfschmerzen im Rahmen einer Lumbalpunktion traten diese auch deutlich häufiger auf, als wenn darüber nicht aufgeklärt wurde [31].Unter „Praxis - Optimierung der Erwartung “ sind Möglichkeiten dargestellt, die Wertbeimessung der therapeutischen Maßnahmen zu optimieren [6].

PRAXIS

Optimierung der Erwartung

- Betonung positiver Effekte und guter Wirksamkeit (Verstärkung Placeboeffekt)

- realistische Aufklärung von Nebenwirkungen, ohne diese überzubetonen (Vermeidung Noceboeffekt)

- persönliches und empathisches Aufklärungsgespräch anstatt nur schriftlicher Aufklärungsbögen

- zusätzlich zum Gespräch Bereitstellung von Informationsmaterialien (Broschüren, Filme) zur weiterführenden Eigeninformation des Patienten

- Erklärung pharmakologischer Wirkmechanismen

- optimistischer und positiver Therapeut

- Anknüpfung an positive Vorerfahrungen

- falls vorhanden, Aufarbeiten negativer Vorerfahrungen

Zudem kann der Behandler durch die Art und Weise der Kommunikation (s. „Fallbeispiel - Postoperative Schmerztherapie“) entscheidenden Einfluss auf die Ausprägung des Placeboeffektes nehmen. Durch eine warm-empathische Gesprächsführung lassen sich im Gegensatz zu einer kühl-technischen Art eine ausgeprägte Steigerung der gewünschten Placeboeffekte sowie eine Reduktion bestehender Ängste erzielen [32-35]. Im klinischen Alltag kann man immer wieder beobachten: Nach einem ausführlichen wertschätzenden Gespräch im Rahmen des Akutschmerzdienstes werden deutlich niedrigere Schmerzniveaus angegeben, auch wenn das eigentliche Therapieregime nicht geändert wird.

Barrett und Kollegen haben eine Liste mit Empfehlungen erarbeitet, die Ärzte einsetzen können, um Placeboeffekte zu generieren [36]. Folgende Dinge spielen dabei eine wichtige Rolle:

- Vertrauen erwecken und Stärkung des Arzt-PatientenVerhältnisses

- Anerkennen von individuellen Patientenbedürfnissen und Werten

- Ermutigung und Beruhigung des Patienten

\section{Merke}

Ein optimistischer Therapeut kann im Aufklärungsgespräch Ängste nehmen und eigene Zuversicht auf den Patienten übertragen, um damit Schmerzerwartung und Wertbeimessung der therapeutischen Maßnahmen zu optimieren.

Auch wenn die Studienlage nicht eindeutig ist, empfiehlt die S3-Leitlinie zur Akutschmerztherapie, den Patienten möglichst realitätsnah auf den zu erwartenden postoperativen Schmerzverlauf und die Beeinflussbarkeit von Schmerzen aufzuklären [4]. Das Wissen um die Kontrollierbarkeit erhöht die Schmerztoleranz und kann präoperative Ängste reduzieren. Ob die Aufklärung jedoch einen relevanten und messbaren Einfluss auf den Schmerzverlauf, den Analgetikaverbrauch und schlussendlich auf die Patientenzufriedenheit hat, wird unterschiedlich bewertet.

\section{Einsatz von Placebopräparaten}

Aktuell wird in der S3-Leitlinie zur Akutschmerztherapie der Einsatz von Placebopräparaten ohne Information und Aufklärung des Patienten als ethisch nicht vertretbar angesehen - sofern eine aktive Schmerztherapie möglich ist [4]. Placebos sollen dementsprechend nur im Rahmen kontrollierter klinischer Studien eingesetzt werden.

Interessant für die Zukunft kann hier der Aspekt der Aufklärung über eine Placebotherapie werden. Mehrere Studien konnten bereits zeigen: Auch die Einnahme eines Placebos - mit dem Wissen, dass es sich um ein Placebo handelt (sog. Open-Label-Placebotherapie) - kann zu einer signifikanten Schmerzreduktion führen [37, 38]. So beschrieben Carvalho und Kollegen: Die zusätzliche Einnahme eines auch als Placebo gekennzeichneten Placebos zusätzlich zur Standardtherapie kann bei chronischen Rückenschmerzen zu einer zusätzlichen Schmerzlinderung und Besserung der Mobilität führen [37]. Ob eine Open-Label-Placebotherapie irgendwann auch als Teil 
einer leitliniengerechten Akutschmerztherapie eingesetzt werden kann, lässt sich jedoch derzeit noch nicht absehen. Hier sind sowohl weitere klinische Studien nötig als auch das Führen einer grundlegenden ethisch-moralischen Diskussion.

\section{KERNAUSSAGEN}

- Placebo- und Noceboeffekte sind nicht auf medikamentöse Therapien beschränkt, sondern treten bei jedem Arzt-Patienten-Kontakt und jeglicher medizinischer Intervention auf.

- Allein durch Placebotherapie lässt sich eine Schmerzreduktion um 20-30\% bewirken.

- Placeboeffekte lassen sich neurobiologisch über eine Aktivierung kortikaler Netzwerke im Sinne des deszendierenden schmerzmodulierenden Systems mit vermehrter Ausschüttung endogener Opioide erklären.

- Placeboeffekte generieren sich aus Lernen inklusive klassischer Konditionierung, sozialem Lernen und Kognition inklusive Erwartung. Vorerfahrungen und Wertbeimessung spielen dabei eine entscheidende Rolle.

- Auch im klinischen Alltag können Placeboeffekte gezielt ausgenutzt werden, um den Effekt analgetischer Therapien zu maximieren (additiver Effekt).

- Bereits bei der präoperativen Aufklärung kann die Voraussetzung für eine Maximierung gewünschter (Placebo-)Effekte und Minimierung von Noceboeffekten geschaffen werden. Außerdem lassen sich wahrscheinlich präoperativ bestehende Ängste reduzieren und die Selbstwirksamkeit optimieren.

- Einfache Maßnahmen, wie eine offene Medikamentengabe und Betonung positiver Effekte, können einen signifikanten Vorteil für den Patienten bewirken.

- Patienten sollten dazu angeleitet werden, Analgetika sehr bewusst einzunehmen und sich auf positive Effekte (Schmerzreduktion) zu fokussieren.

- Potenzielle Nebenwirkungen sollten nicht verschwiegen, aber auch nicht überbetont werden, um die Glaubwürdigkeit zu erhöhen, aber Katastrophisierung zu vermeiden und Noceboeffekte möglichst niedrig zu halten.

- Placebopräparate sollten derzeit nur in Studien eingesetzt werden. Eine Therapie mit Placebos kann sonst schnell zum Vertrauensverlust beim Patienten führen, sollte er davon erfahren.

\section{Interessenkonflikt}

Die Autoren geben an, dass keine Interessenkonflikte vorliegen.

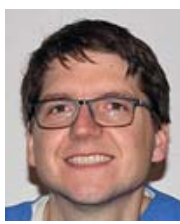

\section{Roland Haaga}

Dr. med., DESA. Seit 2007 in der Klinik und Poliklinik für Anästhesiologie am Universitätsklinikum Hamburg-Eppendorf (UKE). 2010 Zusatzbezeichnung Notfallmedizin, 2013 Facharztprüfung und DESA, 2015 Zusatzbezeichnung Spezielle Schmerztherapie. 2018 Zusatzbezeichnung anästhesiologische Intensivmedizin. Derzeit zu je $50 \%$ in der Schmerzambulanz (Ambulanzzentrum UKE) und in der Klinik und Poliklinik für Anästhesiologie tätig.

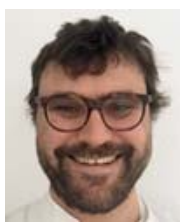

\section{Alexander Schnabel}

PD Dr. med. 2007-2012 Facharztausbildung Anästhesiologie am Uniklinikum Münster, 2013 Zusatzbezeichnungen Intensivmedizin und Notfallmedizin, 2017 Zusatzbezeichnung Spezielle Schmerztherapie. 2014-2018 Facharzt in der Uniklinik Würzburg. Seit August 2018

Facharzt in der Klinik für Anästhesiologie, operative Intensivmedizin und Schmerztherapie am Universitätsklinikum Münster, Mitarbeiter der AG Experimentelle und klinische Schmerzforschung.

\section{Korrespondenzadresse}

\section{Dr. med. Roland Haaga, DESA}

Klinik und Poliklinik für Anästhesiologie

Universitätsklinikum Hamburg-Eppendorf

Martinistraße 52 Gebäude 010

20246 Hamburg

r.haaga@uke.de

\section{Wissenschaftlich verantwortlich} gemäß Zertifizierungsbestimmungen

Wissenschaftlich verantwortlich gemäß Zertifizierungsbestimmungen für diesen Beitrag ist Dr. med. Roland Haaga, Hamburg.

\section{Literatur}

[1] Moseley JB, O'Malley K, Petersen NJ et al. A controlled trial of arthroscopic surgery for osteoarthritis of the knee. $N$ Engl J Med 2002; 347: 81-88

[2] Beard DJ, Rees JL, Cook JA et al. Arthroscopic subacromial decompression for subacromial shoulder pain (CSAW): a multicentre, pragmatic, parallel group, placebo-controlled, threegroup, randomised surgical trial. Lancet 2018; 391: 329-338. doi:10.1016/S0140-6736(17)32457-1

[3] Wartolowska K, Judge A, Hopewell S et al. Use of placebo controls in the evaluation of surgery: systematic review. BM] 2014; 348: g3253. doi:10.1136/bmj.g3253

[4] Klinger R, Thomm M, Bryant M et al. Patienteninformation und -aufklärung. In: Laubenthal H, Becker M, Sauerland S, Neugebauer $\mathrm{E}$, Hrsg. Deutsche interdisziplinäre Vereinigung für Schmerztherapie (DIVS). S3-Leitlinie „Behandlung akuter und perioperativer posttraumatischer Schmerzen“ (2009). AWMF-Reg.-Nr. 001-025 (bis 2013: 041/001), Seiten 15-22. Im Internet: http://www.awmf.org/leitlinien/detail/II/001025.html; Stand: 25.07.2018 
[5] Klinger R. Das Potenzial des analgetischen Plazeboeffektes S3-Leitlinien-Empfehlung zur Behandlung akuter und perioperativer Schmerzen. Anasthesiol Intensivmed Notfallmed Schmerzther 2010; 45: 22-29. doi:10.1055/s-0029-12433

[6] Klinger R, Colloca L, Bingel U et al. Placebo analgesia: clinical applications. Pain 2014; 155: 1055-1058. doi:10.1016/j. pain.2013.12.007

[7] Colloca L, Lopiano L, Lanotte M. Overt versus covert treatment for pain, anxiety, and Parkinson's disease. Lancet Neurol 2004; 3: 679-684

[8] Kelley JM, Lembo AJ, Ablon JS. Patient and practitioner influences on the placebo effect in irritable bowel syndrome. Psychosom Med 2009; 71: 789-797. doi:10.1097/PSY.0b013e3181acee12

[9] Vickers AJ, Vertosick EA, Lewith G et al. Acupuncture for chronic pain: Update of an individual patient Data Meta-Analysis. J Pain 2018; 19: 455-474. doi:10.1016/j.jpain.2017.11.005

[10] Bingel U, Lorenz J, Schoell E et al. Mechanisms of placebo analgesia: rACC recruitment of a subcortical antinociceptive network. Pain 2006; 120: 8-15

[11] Zubieta JK, Yau WY, Scott DJ et al. Belief or Need? Accounting for individual variations in the neurochemistry of the placebo effect. Brain Behav Immun 2006; 20: 15-26. doi:10.1016/j. bbi.2005.08.006

[12] Eippert F, Bingel U, Schoell ED et al. Activation of the opioidergic descending pain control system underlies placebo analgesia. Neuron 2009; 63: 533-543

[13] Eippert F, Finsterbusch J, Bingel $U$ et al. Direct evidence for spinal cord involvement in placebo analgesia. Science 2009; 63: 533-543. doi:10.1126/science. 1180142

[14] Levine JD, Gordon NC, Fields HL. The mechanism of placebo analgesia. Lancet 1978; 2: 654-657

[15] Wager TD, Atlas LY, Lindquist MA et al. An fMRI-based neurologic signature of physical pain. N Engl J Med 2013; 368: 1388-1397. doi:10.1056/NEJMoa1204471

[16] Zubieta JK, Bueller JA, Jackson LR et al. Placebo effects mediated by endogenous opioid activity on mu-opioid receptors. J Neurosci 2005; 25: 7754-7762

[17] Geuter S, Büchel C. Facilitation of pain in human spinal cord by nocebo treatment. J Neurosci 2013; 33: 13784-13790. doi:10.1523/JNEUROSCI.2191-13.2013

[18] Colloca L, Klinger R, Flor $\mathrm{H}$ et al. Placebo analgesia: psychological and neurobiological mechanisms. Pain 2013; 154: 511514. doi:10.1016/j.pain.2013.02.002

[19] Klinger R, Blasini M, Schmitz J et al. Nocebo effects in clinical studies: hints for pain therapy. Pain Rep 2017; 2: e586. doi:10.1097/PR9.0000000000000586

[20] Colloca L, Benedetti F. How prior experience shapes placebo analgesia. Pain 2006; 124: 126-133

[21] Colloca L, Benedetti F. Placebo analgesia induced by social observational learning. Pain 2009; 144: 28-34. doi:10.1016/j. pain.2009.01.033

[22] Müller M, Kamping S, Benrath J et al. Treatment history and placebo responses to experimental and clinical pain in chronic pain patients. Eur J Pain 2016; 20: 1530-1541. doi:10.1002/ ejp. 877

[23] Kessner S, Sprenger $C$, Wrobel $N$ et al. Effect of oxytocin on placeo analgesia: a randomized study. JAMA 2013; 310: 1733-1735. doi:10.1001/jama.2013.277446
[24] Lidstone SC, Schulzer M, Dinelle K et al. Effects of expectation on placebo-induced dopamine release in Parkinson disease. Arch Gen Psychiatry 2010; 67: 857-865. doi:10.1001/archgenpschiatry.2010.88

[25] Waber RL, Shiv B, Carmon Z et al. Commercial features of placebo and therapeutic efficacy. JAMA 2008; 299: 10161017. doi:10.1001/jama.299.9.1016

[26] Tinnermann A, Geuter S, Sprenger $C$ et al. Interactions between brain and spinal cord mediate value effects in nocebo hyperalgesia. Science 2017; 358: 105-108. doi:10.1126/science.aan 1221

[27] Vase L, Petersen GL, Riley JL et al. Factors contributing to large analgesic effects in placebo mechanism studies conducted between 2002 and 2007. Pain 2009; 145: 36-44. doi:10.1016/j. pain.2009.04.008

[28] Amanzio M, Pollo A, Maggi G et al. Response variability to analgesics: a role for non-specific activation of endogenous opioids. Pain 2001; 90: 205-215

[29] Benedetti F. Placebo analgesia. Neurol Sci 2006; 27: S100S102

[30] Sipilä RM, Haasio L, Meretoja T] et al. Does expecting more pain make it more intense? Factors associated with the first week pain trajectories after breast surgery. Pain 2017; 158: 922-930. doi:10.1097/j.pain.000000000000000859

[31] Daniels AM, Sallie R. Headache, lumbar puncture, and expectation. Lancet 1981; 1: 1003

[32] Varelmann D, Pancaro C, Cappiello EC et al. Nocebo-induced hyperalgesia during local anesthetic injection. Anesth Analg 2010; 110: 868-870. doi:10.1213/ANE.0b013e3181cc5727

[33] Kaptchuk T], Kelley JM, Conboy LA et al. Components of placebo effect: randomised controlled trial in patients with irritable bowel syndrome. BM] 2008; 336: 999-1003. doi:10.1136/bmj.39524.439618.25

[34] Verheul W, Sanders A, Bensing J. The effects of physicians' affect-oriented communication style and raising expectations on analogue patients' anxiety, affect and expectancies. Patient Educ Couns 2010; 80: 300-306. doi:10.1016/j. pec.2010.06.017

[35] Di Blasi Z, Harkness E, Ernst E et al. Influence of context effects on health outcomes: a systematic review. Lancet 2001; 357: 757-762

[36] Barrett B, Muller D, Rakel D et al. Placebo, meaning and health. Perspect Biol Med 2006; 49: 178-198. doi:10.1353/ pbm.2006.0019

[37] Carvalho C, Caetano JM, Cunha L et al. Open-label placebo treatment in chronic low back pain: a randomized controlled trial. Pain 2016; 157: 2766-2772

[38] Kam-Hansen S, Jakubowski M, Kelley JM et al. Labeling of medication and placebo alters the outcome of episodic migraine attacks. Sci Transl Med 2014; 6: 218ra5. doi:10.1126/scitranslmed.3006175

\section{Bibliografie}

DOI https://doi.org/10.1055/s-0043-121684 Anästhesiol Intensivmed Notfallmed Schmerzther 2018; 53: 579-590 @ Georg Thieme Verlag KG Stuttgart · New York ISSN 0939-2661 


\section{Punkte sammeln auf CME.thieme.de}

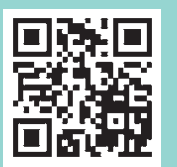

Diese Fortbildungseinheit ist 12 Monate online für die Teilnahme verfügbar.

Sollten Sie Fragen zur Online-Teilnahme haben, finden Sie unter cme.thieme.de/hilfe eine ausführliche Anleitung. Wir wünschen viel Erfolg beim Beantworten der Fragen!

Unter eref/thieme.de/ZZX94GT oder über den QR-Code kommen Sie direkt zum Artikel zur Eingabe der Antworten.

VNR 2760512018154651053

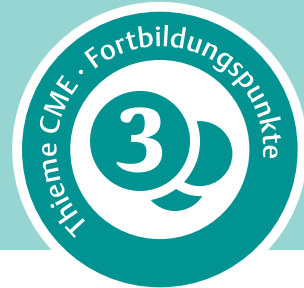

\section{Frage 1}

Welche Aussage zu den Definitionen von Placebo bzw. Nocebo ist richtig?

A Unter Placeboanalgesie versteht man lediglich die Medikamenteneinnahme.

B Ein Placebo gleicht dem Verum in Aussehen und Geschmack.

C Ein Placebo enthält einen spezifischen Wirkstoff.

D Arztgespräche können keinen Placeboeffekt erzielen.

E Eine Placeboanalgesie kann nur in Zusammenhang mit Medikamentengaben genutzt werden.

\section{Frage 2}

Welche Aussage zur Vermittlung placeboanalgetischer Effekte im Endorphinsystem ist richtig? Die Placeboanalgesie wird über...

A Serotonin und entsprechende Rezeptoren vermittelt.

B Dopamin und entsprechende Rezeptoren vermittelt.

C Noradrenalin und entsprechende Rezeptoren vermittelt.

D Opioide und entsprechende Rezeptoren vermittelt.

E Histamin und entsprechende Rezeptoren vermittelt.

\section{Frage 3}

Welche 3 psychologischen Mechanismen werden für das Entstehen eines Placeboeffektes verantwortlich gemacht?

A klassische Konditionierung, soziales Lernen, Erwartungstheorie

B Fear-avoidance, klassische Konditionierung, Selbstachtsamkeit

C Selbstachtsamkeit, soziales Lernen, klassische Konditionierung

D operante Konditionierung, Fear-avoidance, Erwartungstheorie

E klassische Konditionierung, Katastrophisierung, Angstsensitivität

\section{Frage 4}

Welche Aussage zu psychologischen Mechanismen zur Entstehung von Placeboeffekten ist richtig?

A Bei der klassischen Konditionierung wird eine bedingte Reaktion durch eine unbedingte Reaktion ausgelöst.

B Soziales Lernen produziert stärkere Placeboeffekte als klassische Konditionierung.

C Placeboresponder mit schlechten Erfahrungen sprechen auch schlechter auf Placebopräparate an.

D Im Rahmen von Arzt-Patient-Gesprächen kann die Erwartung von Patienten gesteuert werden.

E Über Katastrophisierungsverhalten kann ein Placeboeffekt vermittelt werden.

\section{Frage 5}

Welche Aussage zu Einflussfaktoren auf den Placeboeffekt ist richtig?

A Die Wertbeimessung beeinflusst den Placeboeffekt unabhängig von der Situation.

B Alle Patienten sprechen in ähnlicher Weise auf Placebos bzw. Nocebos an.

C Der therapeutische Kontext kann die Wirkung eines Placebos beeinflussen.

D Lediglich die Beschaffenheit des Placebos ist ein entscheidender Faktor für die Wirkung eines Placebos.

E Bei Patienten mit ausgeprägten Nebenwirkungen in der Vorgeschichte kann kein Placeboeffekt erzielt werden.

\section{Frage 6}

Wodurch zeichnet sich ein gutes Placebo aus?

A invasive Verabreichung

B mittlere Größe des Placebopräparates

$C$ angenehmer Geschmack

D Ausgabe in besonders geringer Zahl

E rosa Farbe

- Weitere Fragen auf der folgenden Seite... 


\section{Punkte sammeln auf CME.thieme.de}

Fortsetzung $\ldots$

\section{Frage 7}

Welche Aussage zur klinischen Anwendung des Placeboeffektes ist richtig?

A Medikamente sollten in verschlossenen Schachteln gegeben werden.

B Medikamente sollten wertneutral verabreicht werden.

C Der Patient sollte die Tablette für die Analgesie nicht kennen.

D Der Patient erhält einen tagesaktuellen Medikamentenplan.

E Die Tabletten sollten alle gleich schmecken.

\section{Frage 8}

Welche Aussage zur klinischen Anwendung des Placeboeffektes ist richtig?

A Die Beschäftigung des Patienten mit der Schmerzintensität und der Schmerzreduktion mindert den Placeboeffekt.

B Die Medikamentengabe sollte nicht zelebriert werden.

C In der Aufklärung sollte auf die Vorerfahrungen des Patienten eingegangen werden.

D Nebenwirkungen von Analgetika sollten mit einem besonderen Gewicht während der Aufklärung besprochen werden.

E Um den Patienten nicht zu verunsichern, sollte die postoperative Schmerztherapie nicht angesprochen werden.

\section{Frage 9}

Welche Aussage zur klinischen Anwendung des Placeboeffektes ist richtig?

A Nebenwirkungen eines Präparates sollten möglichst nicht genannt werden, um einen Noceboeffekt zu vermeiden.

B Eine hohe Schmerzerwartung des Patienten führt zu einer geringeren postoperativen Schmerzintensität.

C Je größer die Versprechungen des Behandlers bezüglich der Analgesie sind, umso größer ist der analgetische Effekt.

D Eine bewusste Wahrnehmung der Schmerzreduktion ist bedeutsam für den Placeboeffekt.

E Die Medikamente sollten besser mithilfe eines Tropfenzählers statt einer PCA-Pumpe gegeben werden.

\section{Frage 10}

Welche Aussage zum Einsatz von Placebopräparaten ist richtig?

A Es ist sinnvoll, Patienten ohne deren Wissen Placebopräparate zu verabreichen.

B Wenn Patienten als Placebo gekennzeichnete Placebopräparate einnehmen, wirken sie logischerweise nicht.

C Placebopräparate sind ohne Nebenwirkungen.

D Placebopräparate kommen im klinischen Alltag häufig zum Einsatz.

E Ein Vorenthalten einer aktiven Schmerztherapie ist ethisch nicht vertretbar. 